\title{
Rate Modulated Pacing Sensor Device
}

National Cancer Institute

\section{Source}

National Cancer Institute. Rate Modulated Pacing Sensor Device. NCI Thesaurus. Code C50303.

A sensor for a pacemaker designed to alter the rate of heart stimulation based on the activity level of the subject. 